\title{
Le bio-bibliografie immaginarie di Bolaño e le biografie infedeli di Orecchio come approcci letterari alle verità della storia, della vita
}

Parole chiave: biofiction, biografia infedele, citazione immaginaria, parodia, letteratura sul male, storia, tempo

DOI: $10.4312 /$ ars.11.2.127-138

\section{Parentele letterarie}

Anche se Davide Orecchio non lo avesse dichiarato in modo esplicito, ${ }^{1}$ il fatto che sulla sua formazione letteraria abbia avuto un peso, tra altre letture, pure quella dell'opera di Bolaño viene suggerito dalle caratteristiche stesse del suo lavoro. Vanno in quel senso tanto una pratica della scrittura quale attività che cresce raccogliendo e travestendo altra scrittura (in un pullulare di richiami intertestuali), quanto un confrontarsi con la storia attraverso il frequente uso di documenti d'invenzione. A parte un riconosciuto debito di entrambi nei confronti di Borges, a legittimare un accostamento dell'opera di questo narratore contemporaneo italiano a quella di Roberto Bolaño, che è stato un autore culto per molti della generazione formatasi nell'ultima fase del XX secolo, non sono, del resto, solo alcune strategie narrative, ma anche un insistito sguardo sulla presenza del male nell'esistenza umana. Si tratta in ogni caso di punti di convergenza che non tolgono nulla a specifiche identità di stile e a precise posizioni di poetica.

Qui s'intende rilevare come entrambi questi scrittori abbiano sfruttato con inventiva il genere biografico proponendo un discorso plurisemantico che è orientato a difendere le ragioni della letterarietà e che sviluppa allo stesso tempo una riflessione critica sulla letteratura e sulla storia. Oggetto di attenzione saranno due testi ben adatti a un confronto, vale a dire: La literatura nazi en América (1996), di Bolaño, e Città distrutte. Sei biografie infedeli (2012), di Orecchio.

1 D. Orecchio (1969) ha una formazione di storico. È scrittore di racconti e giornalista, e s'impone al giudizio della critica con Città distrutte. Nel 2014 pubblica il romanzo Stati di grazia, nel 2017 i racconti Mio padre e la rivoluzione. Per dichiarazioni sulle sue 'paternitä' letterarie cfr. le interviste condotte da E. D’Alessio (2013) e da A. Murtas (2014), dove, fra altri autori, nomina Borges, Bolaño e Kiš. 
Il lavoro del cileno si presenta come un'enciclopedia fittiva della letteratura americana (soprattutto sudamericana) comprendente delle 'schede' bio-bibliografiche della lunghezza di qualche pagina con i dati sulla vita e l'opera di una trentina di scrittori e scrittrici di diversa estrazione sociale, formazione e fortuna. Tutti costoro avrebbero abbracciato l'ideologia nazista o in qualche modo vi avrebbero simpatizzato, facendone spesso anche oggetto di scrittura. Di questi "personaggi nefasti», come li ha definiti una studiosa (Bragado, 2012, 45, n. 6), il narratore calato nei panni di biografo ed esperto di letteratura, avanza anche dei dati sulla genesi e struttura delle loro opere: ne menziona titoli, motivi, stile, fonti e ricezione, ed esprime talvolta anche le proprie considerazioni critiche, ma sempre limitandosi ai testi. Poiché tali giudizi sono formulati per lo più come comparazioni orientate a evidenziare differenze o analogie rispetto a una produzione letteraria di fatto esistente nella realtà e a mostrare influssi, casi d'imitazione e di plagio, il testo stila fra le sue pagine, in modo un po' più nascosto, anche una seconda enciclopedia della letteratura a dimensione mondiale e niente affatto fittiva.

La raccolta di Orecchio comprende, invece, solo poche biografie e di una certa estensione. In questo caso la creazione dei personaggi e del loro percorso di vita cresce tenendo conto delle vicende di alcune persone storicamente esistite, di orientamento impegnato, attive vuoi nell'ambito della politica, vuoi in quelli della letteratura e dell'arte: da qui il sottotitolo pertinente che esclude subito la natura totalmente fittiva di questi racconti biografici. La lettura rileverà poi che la dichiarata 'infedeltà' si attua nei termini di una schermatura, senza risolversi però totalmente in questo procedimento. A collegare i sei soggetti di cui si parla in Città distrutte è soprattutto un percorso biografico che li porta, a un certo punto dell'esistenza, a ritrovarsi consumati interiormente al punto da ridursi nella maggioranza dei casi perfino al silenzio. La biografia diventa allora qui fondamentalmente l'occasione per un'amara riflessione sul tempo, sulla storia e sul senso dell'esistere. ${ }^{2}$

Diversamente che nell'enciclopedia stilata da Bolaño, dove il dato asserito per certo prevale di gran lunga sulla supposizione, nei sei pezzi di Orecchio la congettura s'impone sovrana nel discorso. In tal caso il sapere riferito, fatto di testimonianze in parte attestate, in parte immaginarie, resta aperto all'interpretazione di chi legge e va a sostituire quel sapere trasmesso con certezza che costituiva parte saliente di un tipo di biografia con velleità oggettive, basata su fatti provati e non su congetture, e soprattutto orientata a non lasciare al lettore domande aperte. Più che asserire qualcosa sui suoi biografati, il narratore manifesto di Città distrutte li guarda da vicino, costruisce supposizioni e si prende lo spazio per riflessioni che condivide con i lettori. Non di rado,

2 Sono riprese qui sinteticamente alcune osservazioni espresse in un altro mio articolo su Orecchio. (Cfr. Farinelli, 2017, 135-158) 
sfruttando ampiamente strategie di metalessi, si cala anche come osservatore invisibile in alcuni momenti della loro vita quotidiana e ce li restituisce in quella veste dimessa e più intima. Inutile sottolineare che pure qui l'invenzione è esuberante, ma ancora più ingegnoso è il lavoro di nascondimento e svelamento dei dati che costruiscono il discorso - ed è un procedimento che attiva fortemente il lettore.

\section{Biografia inventiva, biografia infedele}

Entro una produzione testuale con ambizioni letterarie, anche prima del postmoderno, e per tutto un secolo, il genere biografico aveva già trovato talvolta realizzazione in forme tese ad abbatterne i tradizionali criteri di scrittura oggettiva e documentata, e a liberarsi dalle usuali finalità informative e celebrative (aspetti che resteranno invece ancora visibili nella produzione biografica di largo consumo). Lavori come La literatura nazi en América e Città distrutte sono esempi relativamente recenti di una produzione biografica acanonica e tendenzialmente inventiva, riscontrabile già nelle Vies imaginaires di Schwob $(1886)^{3}$. Bolaño era ben consapevole della sfida letteraria lanciata da questo scrittore modernista di lingua francese, se anche in un articolo per Quimeras e poi di nuovo nel prologo ai propri Cuentos, fornendo consigli a narratori che volessero scrivere bene, raccomandava appunto di leggerne l'opera, che riconosceva stare su una linea, lungo la quale si sarebbe arrivati a Borges (Bolaño, 1998, 66; 2010, 8). La sperimentazione intorno al genere biografico s'intensifica, però, decisamente negli ultimi vent'anni del ventesimo secolo, e gli studiosi del genere ne prendono atto. ${ }^{4}$ Domenique Viart, ad esempio, rileva come la biografia sia divenuta, a partire dagli anni ' 80 del Novecento, un genere trasversale tendente a contaminarsi con la narrativa d'invenzione, l'autobiografia e l'essai. (Viart, 2001, 338) Fra le varianti di biografie miste, in questi ultimi decenni trova particolare frequentazione la biofiction, una forma orientata a mantenere aperta un'ambiguità tra dato storico e dato d'invenzione. Riccardo Castellana, rivedendone in modo più preciso le definizioni già avanzate da altri studiosi, la circoscrive come segue:

Ciò che la distingue dalla biografia propriamente detta è l'ibridazione del discorso fattuale (biografico) con tratti testuali della fiction, sia a livello tematico [...], sia a livello formale (tramite l'adozione di dispositivi tipicamente funzionali come la psiconarrazione o l'indiretto libero). L'ibridazione può riguardare, inoltre, anche l’aspetto pragmatico, allorché il carattere di

3 Parlando della genesi de La literatura nazi en América, in un'intervista del 2005 (confluita poi in Braithwaite, 2006, 42), Bolaño ricorda i suoi debiti verso Wilcock (La sinagoga de los iconoclastas) e Borges (Historia universal de la infamia); ricostruendo poi una catena di successivi influssi, osserva che su quest'ultimo avrebbe avuto un peso Alfonso Reyes (Retratos reales e imaginarios), il quale a sua volta sarebbe stato debitore a Schwob (Lòpez de Abiada, J. M., 2012, 15, n. 8). 
finzionalità del testo emerga non tanto dalle caratteristiche interne ad esso (formali o tematiche) quanto piuttosto dalla contraddizione tra queste e la natura del contratto di lettura prospettato fittivamente, specie già a partire dal paratesto. (Castellana, 2015, 70)

Se si segue la definizione sopra riportata, le due opere qui prese in esame rientrano con una certa difficoltà sotto la categoria di biofiction perché non si presentano fondate su fenomeni di vera e propria ibridazione ${ }^{5}$ (nel caso di Città distrutte lo rileva lo stesso Castellana). Mentre la raccolta di Bolaño appartiene, infatti, alle biografie d'invenzione - ma di un'invenzione calata sullo sfondo di contesti storici reali - così che al lettore s'insinua il dubbio che la fantasia non saturi l'intero e che il discorso restituisca forse anche degli eventi accaduti -, quella di Orecchio è impostata su un principio di approssimativo raddoppiamento. Per quanto i biografati di Città distrutte portino nomi d'invenzione e molti dati con cui si costruiscono le loro vite siano immaginari, dietro a tali costruzioni stanno, a parte un caso, delle precise persone storiche. Tra il personaggio letterario e la persona esistita, che vi fa da referente, non viene a crearsi mai, però, una semplice sovrapposizione. Persona storica e personaggio mantengono delle vite parallele, il che è di per sé ovvio quando la realtà storica si fa letteratura, solo che qui lo scarto viene ostentato e rientra in quell'elemento di riflessione metanarrativa sulle leggi del letterario che tutto il testo possiede. Come lautore stesso spiega in uno dei sei pezzi della raccolta, il suo personaggio anche in quel caso «è una possibilità» della figura storicamente esistita (Orecchio, 2012, 235).

Nella consapevolezza che l'opera artistica e letteraria non epigona spiazza sempre ogni tentativo di classificazione, piuttosto che cercare una più precisa nicchia tipologica per i due lavori in questione, merita considerare le sfide e significati aperti dalle loro complesse tessiture.

\section{Raccontare la seduzione del male}

La literatura nazi en América si costruisce su un contrasto stridente tra un tema scabroso come quello della capacità di presa, su certi soggetti, di un'ideologia improntata a violenza e autoritarismo, e le forme retorico-discorsive adottate per parlarne, le quali puntano su un'assenza di giudizio etico e su una prospettiva discorsiva di oggettiva distanza che assume evidente funzione parodica. Come già osservato,

5 Lo stesso studioso specifica che nella biofiction non rientrerebbero: la biografia quale discorso fattuale, l'autobiografia, l'autofiction, la faction (indagine su un certo fatto, che viene sviluppata attraverso un discorso inglobante allo stesso tempo invenzione e cronaca), il romanzo che simula la struttura di una biografia, il romanzo storico, la «fiction basata sulla storia di un personaggio reale i cui protagonisti portano però un nome diverso da quello del modello», e infine altri generi di narrativa finzionale «svincolati dalla prova della referenzialità.» (Castellana, 2015, 72-73) 
i commenti che emergono nelle schede su questi pretesi scrittori sono unicamente indirizzati a giudicarne la qualità stilistica delle opere e i possibili influssi. I rilievi critici atribuiti ad altri, come pure quelli avanzati dalla stessa figura del compilatore delle schede, sono espressi con formule tese a parodiare il codice tipico della critica letteraria. Vediamo costui, ad esempio, in veste di esperto di letteratura, riferire che il romanzo La minestra dei poveri di Ernesto Pérez Masón era formulato «in uno stile impeccabile che avrebbe avuto l'approvazione di Šolochov» (Bolaño, 2013, 69) ${ }^{6}$ o ricordare che, nel caso di Andés Cepeda Cepeda 'El Doncel', i primi tredici poemetti "composti in endecasillabi un tantino confusi lasciarono indifferente la critica» (2013, $82)^{7}$. Ed eccolo, ancora, giudicare il secondo libro di poesie di Franz Zwickau come un libro «maledetto, raccapricciante, mal scritto», o individuare nella raccolta poetica di Argentino Schiaffino «una sfortunata imitazione di Lugones e Darío che in alcuni passaggi giunge al plagio puro e duro [...].» $(2013,103 \text { e 185 })^{8}$ Per averne ancora un esempio basterebbe leggere la scheda in cui si è informati della fortuna riscossa da unopera del preteso scrittore brasiliano e studioso di filosofia, Luiz Fontaine da Da Souza:

Nel 1939 sorprende tutti con la pubblicazione di un breve romanzo sentimentale. Nelle sue scarse 108 pagine (un'altra sorpresa) narra le pene d'amore di un professore di letteratura portoghese per una giovane ricca e pressoché analfabeta di Novo Hamburgo. Il romanzo, Lotta fra gli opposti, vende pochissimo, ma lo stile raffinato, lo spirito brillante e la perfetta economia verbale con cui è costruito non passano inosservati ad alcuni critici che lo elogiano senza riserve. $(2013,64)^{9}$

Nel passo citato risalta, oltre alla formula che imita quelle di tante recensioni letterarie («lo stile raffinato, lo spirito brillante e la perfetta economia verbale»), pure la scelta tra il ludico e l'ironico del titolo del romanzo, Lotta fra gli opposti, in cui echeggia l'amore che questo letterato avrebbe avuto per Hegel. Si tratta di una prassi non priva di divertissement, ritrovabile anche nel caso di altri immaginari testi menzionati nel lavoro, e consistente appunto nel fare passare gli interessi e le manie di questi scrittori 'di carta' nelle scelte dei titoli e dei generi delle opere che si viene

6 «[... en un impecable estilo que hubiera aprobado Sholojov [...].» (Bolaño, 2017, 58)

7 «Los trece poemas de El Destino de la calle Pizarro, compuestos en endecasílabos un tanto confusos, dejaron a la crítica indiferente [...].» (Bolaño, 2017, 71)

8 «Libro maldito, espeluznante, mal escrito» (Bolaño, 2017, 91); «una desafortunada imitación de Lugones y Darío que en ocasiones lega al plagio puro y duro [...].» (Bolaño, 2017, 172)

9 «En 1939 sorprende a todo el mundo con la publicación de una novelita sentimental. En sus escasas 108 páginas (otra sorpresa) narra los requiebros amorosos de un profesor de literatura portuguesa por una joven rica y casi analfabeta de Novo Hamburgo. La novela, Lucha de contrarios, apenas se vende pero su fino estilo, su agudeza y la perfecta economía verbal con que está construida non pasan desapercibidas para algunos críticos que la alaban sin reservas.» (Bolaño, 2017, 54) 
loro attribuendo. La smaccata imitazione del biografo esperto di letteratura, assieme ai numerosi rimandi a testi di fatto esistenti e a ostentati richiami alle pratiche di ripresa, calco, falsificazione che connoterebbero le opere menzionate, è un aspetto centrale del discorso. Oltre a proporsi come operazione metanarrativa attraverso la quale il testo svela i suoi procedimenti, esso finisce per riproporre anche la tesi, popolare in epoca postmoderna, dell'impossibile autenticità della parola. Scrivere tramite formule di largo uso - e qui specificamente quelle della critica - sarebbe allora un modo per evidenziare il rapporto opaco fra l'oggetto e il discorso che ne riferisce, e che si presuppone nato sempre nel calco di altri discorsi. Parallelamente, e in collegamento a tale tesi, emerge nel lavoro del cileno anche un'idea della letteratura come uno spazio dove ogni inizio è sempre un ritorno, una ripresa, una geminazione. Poe, Kafka, Verne, Góngora, Rabelais, Dante sono, ad esempio, solamente alcuni fra i numerosi autori che vengono nominati come fonti di questa immaginaria produzione d'impronta filonazista. Strategia che fa riflettere, dietro il figurale di racconti bio-bibliografici, come la parola nel processo di ricezione prenda svolte inattese e, decontestualizzata e usata arbitrariamente, possa anche alimentare opere ideologicamente turpi.

Entrambe le scelte discorsive rilevate, tanto un discorso di neutralità protocollare, privo di prese di posizione e di qualsiasi riflessione storica, filosofica o morale sui fatti che segnerebbero quelle vite, quanto il registro parodico (due scelte che rispondono rispettivamente alle funzioni di fingere uno stile storiografico e di denudare tale operazione), possono essere sentite come irritanti rispetto al tema affrontato. Un tale procedere è leggibile come strategia di straniamento, tesa a richiamare attenzione su qualcosa che si preferirebbe non vedere, vale a dire la possibilità che il fascino per il male e la sua pratica tocchino chiunque e che il mondo della creazione estetica vi resti coinvolto. Qui lo straniante non si presenta tanto nell'elemento dell'eccesso, che pur è ben presente nel testo sia a livello tematico (negli oggetti e finalità di quella produzione) sia a livello di struttura (nei principi di varietà e moltiplicazione attivati dall'autore nel suo discorso), quanto piuttosto in un récit in cui il tragico e l'abominevole vengono affrontati in modo distante e neutrale, secondo un ductus discorsivo che imita, corrodendolo con ironia, quello tradizionalmente atteso nella trattatistica e in studi accademici. La figura del compilatore de La literatura nazi en América adotta uno sguardo selettivo che sorvola su ciò che urgerebbe di essere focalizzato, per soffermarsi invece con pedanteria su dettagli banali, come il numero di pagine di una data opera. La strategia di un'assente presa di posizione è percepibile forse al meglio nella vicenda conclusiva, vertente sulla vita e l'opera di Carlos Ramírez Hoffman, la cui vicenda trova elaborazione in modo un po' diverso rispetto alle precedenti bio-bibliografie. Bolaño avrebbe non a caso definito quell'ultimo pezzo «un anticlimax» (1996, 11). Nella storia concernente la vita e il lavoro di Carlos Ramírez Hoffman entra lo stesso autore, 
quale personaggio, e in quel ruolo è disposto ad accettare un compenso economico per una precisa collaborazione. Il testo dice in tal caso troppo poco perché il lettore possa ricostruire nei dettagli unopera particolarmente scabrosa del preteso poeta, ma abbastanza per coglierne lorrore. Pienamente condivisibile allora il giudizio di Danuska Gonzáles: riferendosi proprio al lungo racconto Estrella distante, nato come rielaborazione della scheda in questione, questa osservava, appunto, come Bolaño fosse capace di elaborare una lingua del male, e lo facesse puntando sulla sottrazione, sul non detto, attraverso una tecnica orientata ad alludere piuttosto che a descrivere (Gonzáles, 2012, 31).

A riflettere sulle ragioni del fascino del male su chi opera nel mondo della creazione letteraria fu anche, come noto, Georges Bataille. Studiando il caso di otto scrittori e rappresentanti del pensiero della modernità, Bataille comprendeva la loro seduzione per il male come una forma cosciente di libertà, che era poi lo stesso obiettivo perseguito da costoro nell'ambito dell'attività estetica. E precisava: «cette conception ne commande pas l'absence de morale, elle exige une 'hypermorale.» $(1957,8)$ Il discorso di Bolaño è tuttavia diverso. I personaggi da lui creati non rappresentano soggetti dello stesso calibro e con gli stessi obiettivi di personalità quali, ad esempio, i poeti maledetti. ${ }^{10}$ Stanno al contrario per individui che arrivano a simpatizzare quasi banalmente per un'ideologia, e nello specifico per un'ideologia dell'orrore. Rappresentano inoltre le identità più svariate: il soggetto che cerca riscatto e quello che non ne ha per nulla bisogno, l'ingenuo e l'opportunista, il fervido credente e lo scaltro senźanima. Nei rapporti interpersonali taluni coprono persino il ruolo di vittime. In breve, nella rete del male, a seguire la logica attivata nel testo, non entrano solo i carnefici.

Opera scomoda La literatura nazi en América perché, prendendo a emblema unattività reputata pulita come la letteratura, il suo autore solleva la questione del coinvolgimento e delle responsabilità della cultura in ideologie e pratiche eticamente condannabili e, più in generale, del coinvolgimento e delle responsabilità del soggetto che si crede estraneo a ogni connessione con tale ordine di cose. Non è fuori luogo ricordare che, in un'occasione congressuale, Bolaño espresse la sua preoccupazione sulla posizione ambigua di una nuova generazione di scrittori, pronta ad accettare facilmente le leggi del mercato librario per imporsi allattenzione del pubblico e vendere. In termini figurali scriveva: «En realidad somos como niños atrapados en la mansión de un pedófilo. Alguno de ustedes dirá que es mejor estar merced de un pedófilo que a merced de un asesino. Sí, es mejor. Pero nuestros pedófilos son también asesinos.» (Bolaño, 2003, 21)

10 Basti come esempio il capitolo sulla bizzarra vita del preteso poeta sperimentale Willy Schürholz (cfr. Bolaño, 2017, 94-99). 


\section{Raccontare l'azione distruttiva del tempo}

Il paradosso domina certamente anche nelle vite narrate in Città distrutte, ma ben diverso è il modo in cui vi sono rappresentate contraddizioni, debolezze, svolte esistenziali. Invece di parodiare il biografo canonico, che si limita a delineare i fatti, quello di Orecchio segue unattitudine interrogante e apre di continuo delle riflessioni su quanto riferisce: le pratiche d'imitazione vi si realizzano semmai come pastiche dello stile diaristico, della lettera, del saggio letterario e filosofico. A guidare il discorso del biografo di quei sei pezzi, in un gioco esuberante di rimandi intertestuali veri e falsi, è la volontà di comprendere le vite di cui parla, piuttosto che conoscere l'elenco degli eventi che le costruiscono. Con simili scelte narrative la scrittura biografica di Orecchio viene allora a intrecciarsi in modo forte con l'essai. Più il biografo di Città distrutte si pone domande e adduce documenti e opinioni altrui che possano rispondervi, e più si rafforza nella convinzione che comprendere una vita - i suoi moti e le sue svolte - sia impresa impraticabile. Resteranno molti vuoti. Procede allora per ipotesi che lascerà aperte, senza cercare a tutti i costi delle spiegazioni nei casi in cui le ragioni e il senso di una data vicenda biografica (inattese svolte progettuali o contraddizioni di comportamento, ecc.) gli paiono di difficile comprensione. Piuttosto ne fa materia per una riflessione sovra-individuale che finisce per coinvolgere direttamente anche noi lettori. Si chiede ad esempio: «[...] quali regole nascoste ci costringano a naufragare, ad arenarci come relitti indipendentemente dalla nostra forza spirituale.» (Orecchio, 2012, 95) In breve, la serie di racconti biografici raccolta in Città distrutte è sostenuta da una visione epistemologica conscia della parzialità di quanto si conosce. E però una qualche certezza s'insinua fra quegli interrogativi, ed è amara; poggia sull'idea che il tempo agisca sulle esistenze, divorandole. Una simile posizione resta suggerita dal titolo stesso della raccolta, che è tratto da un diario autentico. Il tempo, allora, risulta per lo più associato, in questo caso, a immagini che mostrano la vita umana consumarsi come una cosa. Lo scorrere dei giorni, mai contato con le lancette, bensì nel modo in cui il soggetto lo vive, vi appare non a caso come un involucro vuoto che, fatto il suo uso, è da buttare. Nel racconto biografico del personaggio che rappresenta una sorta di 'doppio' del padre dell'autore, quale persona storica, si legge allora: «Altro tempo come scatole di cartone vuote accostate alla porta per la spazzatura. Migliorisi lo butta via in fretta [...].» $(2012,143)$ E poi ancora, nella biografia di Kaudar (figura approssimativa di Wilhelm von Humboldt): «Lui è il ragno. Nel corso del tempo ha divorato le intenzioni. Adesso gli stanno attorno carcasse, gusci vuoti.» $(2012,209-210)$

Accanto al motivo dellazione distruttiva tempo, vale a dire del veloce dissolversi della storia di una vita e del poco peso che vi ha la memoria, resta centrale in Città 
distrutte anche un discorso sugli orrori che attraversano la storia: si fa parola in particolare di alcuni episodi abominevoli accaduti sotto regimi dittatoriali e violenti, come lo stalinismo, il fascismo e la dittatura argentina. Nel ritornare su simili momenti storici il narratore indugia su particolari cruenti e brutali, mentre, con un uso attento della preterizione, si propone magari di tacerne.

Se un destino di perdita, di vuoto, incombe sulle singole esistenze e un moto di violenza e distruzione regola la storia collettiva, quale allora il compito della scrittura, e di una scrittura operante, nello specifico, con lo strumento dell'infedeltà al vero? Considerando la centralità che nel testo ha l'interrogazione, si direbbe proprio quello di cercare senso anche là dove questo pare assente.

Attraverso un discorso fondato sul dubbio e improntato a una seria riflessione storica ed esistenziale anche là dove l'invenzione apre spazio al ludico, Orecchio condivide con Bolaño (ben più ironico e corrosivo nella scelta di un ductus discorsivo assertivo e pseudo-accademico) la capacità di costruire pagine di eccellente (meta) narrativa sfruttando un genere tradizionalmente più vicino alla storiografia. Se Bolaño denuncia, con la stesura di bio-bibliografie immaginarie, la banalità che porta il singolo al coinvolgimento nel male, dietro semi-schermatura Orecchio focalizza lo sguardo sui mali, anche storici, che svuotano una vita. Il genere biografico, vitalizzato in entrambi i casi da una capacità inventiva che attinge a svariatissime letture e teso a toccare scomode o amare verità dell'umano vivere, raramente ha saputo dire cosi tanto.

\section{Bibliografia}

Bataille, G., La littérature et le mal, Paris 1957.

Bolaño, R., Números, Quimera, 166, 1998, p. 66.

Bolaño, R., Sevilla me mata, in: Palabra de América, Barcelona 2003, pp. 17-21.

Bolaño, R., Cuentos, Barcelona 2010.

Bolaño, R., La letteratura nazista in America (trad. ital. di Nicola, M.), Milano 2013.

Bolaño, R., La literatura nazi en América, Barcelona 2017.

Bragado, M. J. B., El poeta-detective-asesino, personaje recurrente y alterego en la narrativa de Roberto Bolaño, in: Roberto Bolaño. Estrella cercana, Ensayos sobre su obra (ed. López Bernasocchi, A., López de Abiada, J. M.), Madrid 2012, pp. 41-55.

Braithwaite, A., Bolaño por sí mismo, entrevistas, scogidas, Santiago-Chile 2006.

Castellana, R., La biofiction. Teoria, storia, problemi. Allegoria 70-71, serie III, 2015, pp. 67-97. 
D’Alessio, E., Orecchio, D., Le interviste dei Serpenti: Davide Orecchio, Via dei Serpenti, 2013, http://www.viadeiserpenti.it/le-interviste-dei-serpenti-davide-orecchio [25. 6. 2017].

Farinelli, P., Feconda infedeltà: l'innovativa prospettiva biografica di Davide Orecchio, in: Soliloqui e tradimenti: narrativa italiana tra modernismo e postmoderno, Roma 2017, pp. 135-158.

Gonzáles, D., El silencio del mal, Quimera 241, 2004, pp. 28-31.

Iovinelli, A., Il rinnovamento della biografia in Italia (1970-1995), Studia Romanica et Anglica Zagrabiensa 45-46, 2000-2001, pp. 255-295.

Lòpez de Abiada, J. M., Hacia Bolaño, Una introducción, in: Roberto Bolaño. Estrella cercana, Ensayos sobre su obra (ed. López Bernasocchi, A., López de Abiada J. M.) Madrid 2012, pp. 11-40.

Murtas, A., Davide Orecchio, 2014, https://davideorecchio.files.wordpress. com/2014/07/2014_07-mucchio-orecchio.pdf [25. 6. 2017].

Orecchio, D., Città distrutte, Roma 2012.

Orecchio, D., Stati di grazia, Milano 2014.

Viart, D., «Essais-fictions: les biographies (ré)inventées», in: Léclatement des genres au XXe siècle (ed. Dambre, M., Gosselin-Noat, M.), Paris 2001, pp. 331-345. 


\section{Patrizia Farinelli}

\section{Imaginarne biobibliografije Bolaña in polfiktivne biografije Orecchia kot literarni pristopi $k$ resnicam zgodovine in življenja}

Ključne besede: biofikcija, polfiktivna biografija, namišljeni citat, parodija, literatura in zlo, zgodovina, čas.

Že več kot stoletje, še posebej v postmoderni, je mogoče opaziti tip biografije, ki temelji bodisi na izumu bodisi na stičišču dokumentirane in izmišljene snovi. Usmerjen je tako, da v nedoločenem ohrani razmerje med realnim in imaginarnim. Nadvse primeren je za odkrivanje problematičnih ovir žanra, ki je tradicionalno povezan z objektivnimi ambicijami, hkrati pa odseva identiteto literarnega diskurza. $\mathrm{V}$ prispevku sta obravnavani deli iz zadnjih dvajsetih let, ki sodita v že omenjeno produkcijo: prvo delo, La literatura nazi en América Roberta Bolaña (1996), temelji predvsem na domišljiji; drugo delo, Città distrutte: sei biografie infedeli Davida Orecchia (2012), skozi prakso nezvestobe rekonstruira življenja resničnih ljudi in posebnih zgodovinskih kontekstov (med drugim v času fašizma v Italiji in med diktaturo $\mathrm{v}$ Argentini). Primerjava pokaže na svojevrstne strategije in potencialne namene ter posebej poudari, kako se za iznajdljivimi pripovednimi izbirami (ki so zmožne bralca na napačno sled speljati tudi s parodijo tradicionalne biografske pripovedi) gradi radikalen poziv k zgodovinski zavesti. 
Patrizia Farinelli

\section{The fictitious bio-bibliographies of Roberto Bolaño and the semi-fictitious biographies of Davide Orrechio as literary approaches towards the historical and everyday truth}

Keywords: biofiction, semi-fictitious biography, fictitious reference, parody, literature and evil, history, time

There is one type of biography that maintains an ambiguity with regard to what is true and what is not, being based solely on fiction or a mixture of documented and invented information. Such a form has been used in literature for more than a century, but became especially popular with the rise of postmodernism. With its help it is easier to notice the problematic traits of a traditionally impersonal biography and at the same time reflect on the identity of literary discourse. We are thus going to examine two works, published in the last two decades, which can be considered as examples of such literary production. The first is Roberto Bolaño's La literatura nazi en América (Nazi Literature in the Americas), published in 1996, and based on imaginary facts set in a historically realistic context. The second one, published in 2012, is Davide Orecchio's Città distrutte: sei biografie infedeli, which depicts with fictional biographies the lives of real people and actual historical events (among others, fascism in Italy and dictatorship in Argentina). The analysis reveals some specific strategies and possible aims and, in particular, points out that behind various ingenious narrative choices (capable of misleading the reader with their parody of traditional biographical discourse) there is a radical reference to historical consciousness. 